\title{
Density dependence of quasifree single-nucleon knockout reactions
}

\author{
Jan Ryckebusch, ${ }^{*}$ Wim Cosyn, ${ }^{\dagger}$ and Maarten Vanhalst ${ }^{\ddagger}$ \\ Department of Physics and Astronomy, Ghent University, Proeftuinstraat 86, B-9000 Ghent, Belgium
}

(Received 7 February 2011; published 6 May 2011)

\begin{abstract}
We address the issue of whether quasifree single-nucleon knockout measurements carry sufficient information about the nuclear interior. To this end, we present comparisons of the reaction probability densities for $A\left(e, e^{\prime} p\right)$ and $A(p, 2 p)$ in quasifree kinematics for the target nuclei ${ }^{4} \mathrm{He},{ }^{12} \mathrm{C},{ }^{56} \mathrm{Fe}$, and ${ }^{208} \mathrm{~Pb}$. We adopt a comprehensive framework based on the impulse approximation and on a relativized extension of Glauber multiple-scattering reaction theory in which the medium effects related to short-range correlations (SRCs) are implemented. It is demonstrated that SRCs weaken the effect of attenuation. For light target nuclei, both the quasifree $(p, 2 p)$ and $\left(e, e^{\prime} p\right)$ can probe average densities of the same order as nuclear saturation density $\rho_{0}$. For heavy nuclei such as ${ }^{208} \mathrm{~Pb}$, the probed average densities are smaller than $0.1 \rho_{0}$ and the $\left(e, e^{\prime} p\right)$ reaction is far more efficient in probing the bulk regions than $(p, 2 p)$.
\end{abstract}

DOI: 10.1103/PhysRevC.83.054601

PACS number(s): 25.30.Rw, 25.40.Ep, 24.10.Jv

\section{INTRODUCTION}

Single-nucleon knockout reactions from nuclei in quasifree kinematics continue to be a major source of information of the mean-field properties of nuclei. In a quasifree $A\left(e, e^{\prime} p\right)$ reaction, a bound nucleon in the target nucleus $A$ is subjected to an electron-nucleon interaction and ejected, thereby leaving the residual nucleus in a low-lying hole state. The shape of the measured differential cross sections and the knowledge about the energy of the residual nucleus allow one to determine the quantum numbers of the nucleon that was struck by the virtual photon. Electroinduced single-proton knockout experiments with stable nuclear targets have systematically pointed toward the validity but also the limitations of the mean-field picture for understanding nuclei $[1,2]$.

The measured quasifree $A\left(e, e^{\prime} p\right)$ differential cross sections provided evidence for the mean-field picture in that the extracted momentum distributions for the bound nucleons could be modeled with mean-field single-particle wave functions. It should be stressed that the measured momentum distributions cannot be directly related to the single-particle wave functions in momentum space but are distorted in the sense that they are affected by the attenuation effects of the nuclear medium on the ejected proton. The systematic observation that the extracted spectroscopic factors from $A\left(e, e^{\prime} p\right)$ studies are substantially smaller than the predicted single-particle level occupancies provides evidence for the limitations of the mean-field picture [3]. The extracted spectroscopic factors are defined as the overall normalization factor between the measured and the computed differential cross sections. The computed $A\left(e, e^{\prime} p\right)$ observables are based on models that make assumptions for the electron-nucleus interaction, the nuclear wave functions, and the effect of nuclear attenuation on the ejected proton. The latter effect is often referred to as final-state interactions (FSIs). The credibility of a reaction model for $A\left(e, e^{\prime} p\right)$

\footnotetext{
*Jan.Ryckebusch@UGent.be

†Wim.Cosyn@UGent.be

${ }^{\ddagger}$ Maarten.Vanhalst@UGent.be
}

depends on its ability to describe, for example, the differential cross sections and polarization observables for a number of target nuclei. An interesting question is whether the extracted normalization factors can be related to the shell occupancy in a model-independent fashion [4,5].

An alternative method of gaining access to the mean-field properties of nuclei is the $A(p, 2 p)$ reaction [6]. For the study of stable nuclei one may prefer the $A\left(e, e^{\prime} p\right)$ reaction as it leads to some reduced parameter dependence of the extracted results, such as spectroscopic factors. In the first place, this is attributable to the electromagnetic character of the interaction vertex. Second, for obvious reasons the sensitivity to the modeled nuclear attenuation is larger for $(p, 2 p)$ than for $\left(e, e^{\prime} p\right)$. Electron-scattering experiments from unstable nuclei are a real technical challenge and could, for example, be performed at an $e A$ collider such as the one that is on the drawing table for the ELISe (electron-ion scattering in a storage ring) project at FAIR [7]. In inverse kinematics [i.e., the $p(A, 2 p) A-1$ process] the $A(p, 2 p)$ reaction offers great perspectives for investigating the mean-field properties of unstable nuclei [8]. For example, one of the fundamental questions we need to ask ourselves regards how the singleparticle properties of nuclei evolve as a function of the proton-to-neutron ratio. Recent studies [9] with the heavy-ioninduced nucleon-knockout reactions ${ }^{9} \mathrm{Be}(A, A-1) X$ suggest that the deduced spectroscopic factors for single-proton and single-neutron knockout are dramatically dependent on the asymmetry of the proton and neutron Fermi surface. The ${ }^{9} \mathrm{Be}(A, A-1) X$ process is extremely surface dominated and the above-mentioned results with regard to spectroscopic factors await confirmation with a reaction probe which is more efficient in probing the nuclear interior.

The scientific potential of the $(p, 2 p)$ reaction in inverse kinematics to study the mass number dependence of mean-field properties is nicely illustrated in Ref. [10]. There, results of $(p, 2 p)$ measurements on the eight carbon isotopes ${ }^{9-16} \mathrm{C}$ are presented. The measurements provide empirical information about the mass-number dependence of the weakly bound and inner-shell protons. It is shown that the systematics of the momentum distributions, separation energies, and 


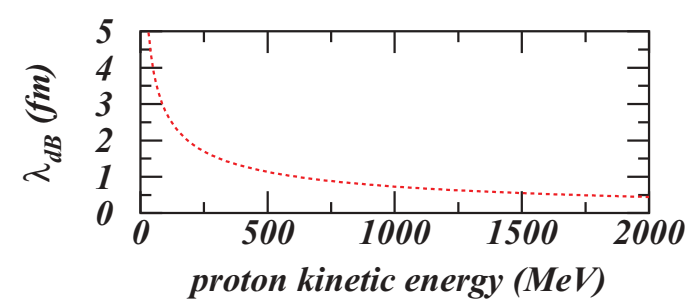

FIG. 1. (Color online) The de Broglie wavelength $\left(\lambda_{\mathrm{dB}}\right)$ of a proton as a function of its kinetic energy.

spectroscopic factors as a function of the mass number can be studied. The $(p, 2 p)$ measurements of Ref. [10] are performed at energies of $250 \mathrm{MeV} / \mathrm{A}$. Quasifree $(p, 2 p)$ measurements for protons of several hundreds of $\mathrm{MeV} / \mathrm{A}$ are part of the research program with high-energy heavy-ion beams at the accelerator complex FAIR [11]. The conditions of high energy are beneficial from the theoretical point of view. First, under high-energy conditions the $(p, 2 p)$ interaction range is small compared to the size of the target nucleus and one can make use of the zero-range approximation when modeling the proton-nucleus interaction vertex. Second, for fast continuum nucleons with a sufficiently small de Broglie wavelength, the effect of attenuation can be appropriately and accurately computed in the semiclassical eikonal approximation [12] even when adopting a Dirac treatment of the nucleons in the initial and final channel [13]. From Fig. 1 it is clear that the proton de Broglie wavelength drops below $1 \mathrm{fm}$ for nucleon kinetic energies larger than about $500 \mathrm{MeV}$. Several studies [14] have pointed toward the applicability of the eikonal method up to remarkably low nucleon kinetic energies of about $250 \mathrm{MeV}$.

Just as for the $A\left(e, e^{\prime} p\right)$ reaction, the observables from $A(p, 2 p)$ measurements are convoluted in that nuclear attenuation does not allow one to relate the measured ejected nucleon properties directly to the physics at the interaction point. With one proton subject to initial-state interactions (ISIs), and two protons subject to FSI, the development of a reliable reaction theory is of the utmost importance for a quantitative analysis of the $A(p, 2 p)$ data $[12,15,16]$. It is well established that nuclear attenuation tends to make the detected signals less sensitive to the high-density regions of the target. Accordingly, one point of concern is whether one may learn something about the bulk properties of nuclei from $A(p, 2 p)$. In other words, do the ejected nucleons carry information about the interior of the system or do they mostly originate from the peripheral regions of the nucleus? In this paper we wish to address this issue. We attempt to quantify what regions of the target nucleus can be probed in $A(p, 2 p)$. For the sake of reference, we also add results for $A\left(e, e^{\prime} p\right)$. The latter serve as a benchmark, as with $A\left(e, e^{\prime} p\right)$ a lot has been learned about single-particle properties of stable nuclei during the last couple of decades. We focus on single-nucleon knockout reactions with nucleon kinetic energies of several hundreds of $\mathrm{MeV}$. At those energies the Glauber multiple scattering framework is both appropriate and accurate to model nuclear attenuation.
In Sec. II A, we outline the necessary formalism for quasifree single-nucleon knockout in a relativistic and crosssection factorized framework. In Sec. II B a relativized version of Glauber multiple scattering theory is introduced and it is pointed out how it can be corrected for the medium effects related to short-range correlations (SRCs). The results of the the numerical calculations are discussed in Sec. III and a summary is given in Sec. IV.

\section{THEORY}

\section{A. Cross sections}

The theoretical calculations for the $A(p, p N)$ and $A\left(e, e^{\prime} N\right)$ reactions presented here are performed with a factorized form for the cross sections. They represent very useful zeroth-order approximations and are often used as a starting point for the interpretation of the data. The factorization is largely based on the impulse approximation (IA), which develops out of a reaction picture in which $(A-1)$ nucleonic degrees of freedom are frozen during the interaction of the external probe and the target. In the IA, the role of the spectator $(A-1)$ nucleonic degrees of freedom is restricted to distorting the waves of the impinging and ejected nucleons. In addition, the factorization allows for a more direct comparison between the $(p, 2 p)$ and the $\left(e, e^{\prime} p\right)$, as their differential cross sections become proportional to a distorted momentum distribution that is related to the probability of finding a nucleon with well-defined quantum numbers and a certain momentum in the target nucleus. First, we derive a factorized expression for the $A\left(e, e^{\prime} p\right)$ and the $A(p, 2 p)$ cross sections.

Consider the $A\left(e, e^{\prime} N\right) A-1$ process and define the corresponding kinematic variables of the impinging electron, the target nucleus, the scattered electron, the residual nucleus, and the ejected nucleon as

$$
\begin{aligned}
K^{\mu}(\epsilon, \vec{k}) & +K_{A}^{\mu}\left(E_{A}, \vec{k}_{A}\right) \longrightarrow K^{\mu}\left(\epsilon^{\prime}, \vec{k}^{\prime}\right) \\
& +K_{A-1}^{\mu}\left(E_{A-1}, \vec{k}_{A-1}\right)+K_{N}^{\mu}\left(E_{N}, \vec{k}_{N}\right) .
\end{aligned}
$$

The fivefold differential cross section in the laboratory frame adopts the form [17]

$$
\frac{d^{5} \sigma}{d \epsilon^{\prime} d \Omega_{e^{\prime}} d \Omega_{N}}=\frac{m_{e}^{2} k^{\prime} k_{N} M_{A-1} M_{N}}{(2 \pi)^{5} \beta_{e A} \epsilon M_{A}}\left(f_{\mathrm{rec}}^{e A}\right)^{-1} \overline{\sum_{i f}}\left|\mathcal{M}_{f i}^{\left(e, e^{\prime} p\right)}\right|^{2}
$$

where $\beta_{e A}=\frac{k}{\epsilon} \approx 1$ is the relative velocity and $\overline{\sum_{i f}}$ corresponds to the appropriate average over initial states and sum over final states. We assume that all wave functions entering the computation of the reaction amplitude $\mathcal{M}_{f i}^{\left(e, e^{\prime} p\right)}$ are normalized to unity. Throughout this work, the adopted normalization convention for the Dirac spinors is $\vec{u}\left(\vec{k}, m_{s}\right) u\left(\vec{k}, m_{s}\right)=1$. The recoil factor $f_{\text {rec }}^{e A}$ in Eq. (2) reads

$$
f_{\mathrm{rec}}^{e A}=\frac{E_{A-1}}{M_{A}}\left[1+\frac{E_{N}}{E_{A-1}}\left(1-\frac{\vec{q} \cdot \vec{k}_{N}}{k_{N}^{2}}\right)\right],
$$

where the momentum transfer has been defined as

$$
\vec{q}=\vec{k}-\vec{k}^{\prime}
$$


For the kinematics of the $A(p, 2 p) A-1$ we adopt the following conventions:

$$
\begin{aligned}
P_{1}^{\mu}\left(E_{p 1}, \vec{p}_{1}\right) & +K_{A}^{\mu}\left(E_{A}, \vec{k}_{A}\right) \rightarrow K_{1}^{\mu}\left(E_{k 1}, \vec{k}_{1}\right) \\
& +K_{2}^{\mu}\left(E_{k 2}, \vec{k}_{2}\right)+K_{A-1}^{\mu}\left(E_{A-1}, \vec{k}_{A-1}\right),
\end{aligned}
$$

where $P_{1}^{\mu}$ refers to the impinging nucleon and $\left(K_{1}^{\mu}, K_{2}^{\mu}\right)$ to the pair of ejected nucleons. The fivefold differential equation for the $A(p, 2 p) A-1$ process can be straightforwardly derived from the $A\left(e, e^{\prime} p\right)$ one [Eq. (2)] by means of the substitutions $m_{e} \rightarrow M_{N}, \epsilon \rightarrow E_{p 1}, k^{\prime} \rightarrow k_{1}, k_{N} \rightarrow k_{2}$ and this results in

$\frac{d^{5} \sigma}{d E_{k 1} d \Omega_{1} d \Omega_{2}}=\frac{M_{N}^{3} k_{1} k_{2} M_{A-1}}{(2 \pi)^{5} \beta_{p A} E_{p 1} M_{A}}\left(f_{\mathrm{rec}}^{p A}\right)^{-1} \overline{\sum_{i f}}\left|\mathcal{M}_{f i}^{(p, 2 p)}\right|^{2}$,

where the recoil factor reads

$$
f_{\mathrm{rec}}^{p A}=\frac{E_{A-1}}{M_{A}}\left[1+\frac{E_{k 2}}{E_{A-1}}\left(1-\frac{\vec{q} \cdot \vec{k}_{2}}{k_{2}^{2}}\right)\right] .
$$

In this expression the momentum transfer is defined as

$$
\vec{q}=\vec{p}_{1}-\vec{k}_{1} \text {. }
$$

In the laboratory frame one obtains the following expression for the relative velocity: $\beta_{p A}=\frac{p_{1}}{E_{p 1}}$.

The squared amplitude $\overline{\sum_{i f}}\left|\mathcal{M}_{f i}^{(p, 2 p)}\right|^{2}$ can be related to the free proton-proton cross section after making several assumptions. They are pointed out in great detail in Ref. [16] and include the neglect of the negative-energy components in the proton-proton scattering amplitude. Further, one assumes that the operator describing the collision of the impinging proton $\vec{p}_{1}$ and the bound proton $\vec{p}_{m}$ resulting can be described in terms of the on-shell proton-proton scattering amplitude. Now we sketch how one can arrive at a factorized expression for the amplitude $\mathcal{M}_{f i}^{(p, 2 p)}$. We denote the position coordinates of the impinging proton as $\vec{r}_{0}$, of the ejected protons as $\left(\vec{r}_{0}, \vec{r}_{1}\right)$, and of the nucleons in the target nucleus as $\left(\vec{r}_{1}, \ldots, \vec{r}_{A}\right)$. In the eikonal approach, the attenuation can be accounted for by means of a multiplicative factor to be applied to a plane-wave wave function. Accordingly, the relativistic distorted wave function of the impinging proton reads

$$
\begin{aligned}
\phi_{\vec{p}_{1}, m_{s 1}}^{D} & =\widehat{S}_{p 1}\left(\vec{r}_{0}, \vec{r}_{2}, \ldots, \vec{r}_{A}\right) \sqrt{\frac{E+M}{2 M}}\left[\begin{array}{c}
1 \\
\frac{\vec{\sigma} \cdot \vec{p}_{1}}{E+M}
\end{array}\right] e^{i \vec{p}_{1} \cdot \vec{r}_{0}} \chi_{\frac{1}{2} m_{s 1}} \\
& =\widehat{S}_{p 1} e^{i \vec{p}_{1} \cdot \vec{r}_{0}} u\left(\vec{p}_{1}, m_{s 1}\right),
\end{aligned}
$$

where $u(\vec{p}, s)$ is a four-component free-particle Dirac spinor. For the distorted wave functions $\phi_{\vec{k}_{1}, m_{s 1}^{\prime}}^{D}$ and $\phi_{\vec{k}_{2}, m_{s 2}^{\prime}}^{D}$ of the ejected protons, similar expressions hold. In the above expression, the $A$-body operator $\widehat{S}_{p 1}$ is fully responsible for the effect of attenuation. In this work, we compute the effect of attenuation in a relativized Glauber model. More details are provided in Sec. II B. The $\widehat{S}_{p 1}$ is a two-by-two matrix which acts on the Pauli spinors. Here we assume that $\widehat{S}_{p 1}$ is a diagonal matrix which amounts to neglecting the spin-dependent attenuation mechanisms. Indeed, the central component of the nucleon-nucleon scattering amplitude accounts for the major impact of nuclear attenuation in high-energy proton-nucleus collisions [18]. Recent investigations [19] have clarified the role of the spin-dependent terms in the nucleon-nucleon scattering amplitude for the attenuation effects in $D\left(e, e^{\prime} p\right) n$. The central component was found to dominate the attenuation for most observables, and in particular for the differential cross sections at low missing momenta. Accordingly, we deem that inclusion of the central component is sufficiently accurate for our current purposes, namely, making a comparative study of the density dependence of the $\left(e, e^{\prime} p\right)$ and $(p, 2 p)$ reaction throughout the mass table.

We now proceed with the derivation of a factorized expression for the $\left(e, e^{\prime} p\right)$ and $(p, 2 p)$ cross sections. In what follows we describe the wave function of the target nucleus by a normalized Slater determinant $\left|\alpha_{1} \alpha_{2}, \ldots, \alpha_{A}\right\rangle$, where the $\alpha_{i}$ refer to the quantum numbers of the occupied single-particle states. With the distorted wave functions of Eq. (9) we get after neglecting the negative-energy projection term the following expression [16]:

$$
\begin{aligned}
\mathcal{M}_{f i}^{(p, 2 p)} \approx & \sum_{s} \int d \vec{r} \widehat{\mathcal{S}}_{\mathrm{RMSGA}}^{(p, 2 p)}(\vec{r}) e^{-i \vec{p} \vec{p}_{m} \cdot \vec{r}} \bar{u}\left(\vec{p}_{m}, s\right) \phi_{n \kappa m}(\vec{r}) \\
& \times\left[u^{\dagger}\left(\vec{k}_{1}, m_{s 1}^{\prime}\right) u^{\dagger}\left(\vec{k}_{2}, m_{s 2}^{\prime}\right) \widehat{F}_{p p} u\left(\vec{p}_{1}, m_{s 1}\right) u\left(\vec{p}_{m}, s\right)\right]
\end{aligned}
$$

where $\widehat{F}_{p p}$ is the $p p$ scattering amplitude in momentum space. The quantum numbers $(n \kappa m)=\alpha_{1}$ determine the orbit of the struck nucleon, which is described by a relativistic singleparticle wave function $\phi_{n \kappa m}(\vec{r})$. The missing momentum $\vec{p}_{m}$ is determined by the difference between the asymptotic three momentum of the ejected nucleon $\vec{k}_{2}$ and the three-momentum transfer $\vec{q}=\vec{p}_{1}-\vec{k}_{1}$ :

$$
\vec{p}_{m}=\vec{k}_{2}+\vec{k}_{1}-\vec{p}_{1}=-\vec{k}_{A-1} .
$$

In the absence of nuclear attenuation, the missing momentum equals the momentum of the bound nucleon with quantum numbers $(n \kappa m)$ which collides with the proton beam. In the above equation (10) we have introduced an operator which accounts for the ISI/FSI [16],

$$
\begin{aligned}
\widehat{\mathcal{S}}_{\mathrm{RMSGA}}^{(p, 2 p)}(\vec{r})= & \prod_{i=2}^{i=A} \int d \vec{r}_{i}\left|\phi_{\alpha_{i}}\left(\vec{r}_{i}\right)\right|^{2} \widehat{S}_{p 1}\left(\vec{r}, \vec{r}_{2}, \ldots, \vec{r}_{A}\right) \\
& \times \widehat{S}_{k 1}\left(\vec{r}, \vec{r}_{2}, \ldots, \vec{r}_{A}\right) \widehat{S}_{k 2}\left(\vec{r}, \vec{r}_{2}, \ldots, \vec{r}_{A}\right)
\end{aligned}
$$

a multidimensional convolution over the squared wave functions of the spectator nucleons and a product of the scalar operators $\widehat{S}_{k}$ for the impinging proton and two ejected protons.

With the aid of Eq. (10) one arrives at the following factorized form for the fivefold $A(p, 2 p)$ differential cross section of Eq. (6):

$$
\begin{aligned}
\frac{d^{5} \sigma}{d E_{k 1} d \Omega_{1} d \Omega_{2}} \approx & \frac{(2 \pi)^{3} s k_{1} k_{2} M_{A-1}}{M_{N} p_{1} M_{A}}\left(f_{\text {rec }}^{p A}\right)^{-1} \\
& \times\left(\frac{d \sigma^{p p}}{d \Omega}\right)_{\text {c.m. }} S_{n \kappa} \rho_{n \kappa}^{D}\left(\vec{p}_{m}\right),
\end{aligned}
$$

where $\left(\frac{d \sigma^{p p}}{d \Omega}\right)_{\text {c.m. }}$ is the center-of-mass cross section for $p p$ scattering at an invariant energy $W=\sqrt{s}$. The $S_{n \kappa}$ is the spectroscopic factor $\left(0<S_{n \kappa} \leqslant 1\right)$ that is related to the 
occupancy of the orbit $(n \kappa)$ in the ground state of the target nucleus. In the above expression the distorted momentum distribution $\rho_{n \kappa}^{D}\left(\vec{p}_{m}\right)$ adopts the form [20]

$$
\begin{aligned}
\rho_{n \kappa}^{D}\left(\vec{p}_{m}\right)= & \sum_{s, m}\left|\int d \vec{r} \frac{e^{-i \vec{p}_{m} \cdot \vec{r}}}{(2 \pi)^{3 / 2}} \bar{u}\left(\vec{p}_{m}, s\right) \widehat{\mathcal{S}}_{\mathrm{RMSGA}}^{(p, 2 p)}(\vec{r}) \phi_{n \kappa m}(\vec{r})\right|^{2} \\
= & \sum_{s, m}\left[\phi_{n \kappa m}^{D}\left(\vec{p}_{m}\right)\right]^{\dagger} \phi_{n \kappa m}^{D}\left(\vec{p}_{m}\right) \\
= & \frac{1}{2} \int d r \int d \theta\left(\sum _ { s , m } \left\{[D(r, \theta)]^{\dagger} \phi_{n \kappa m}^{D}\left(\vec{p}_{m}\right)\right.\right. \\
& \left.\left.+D(r, \theta)\left[\phi_{n \kappa m}^{D}\left(\vec{p}_{m}\right)\right]^{\dagger}\right\}\right) \\
\equiv & \int d r \int d \theta \delta(r, \theta)
\end{aligned}
$$

where

$$
D(r, \theta)=\int d \phi r^{2} \sin \theta \frac{e^{-i \vec{p}_{m} \cdot \vec{r}}}{(2 \pi)^{3}} \bar{u}\left(\vec{p}_{m}, s\right) \widehat{\mathcal{S}}_{\mathrm{RMSGA}}^{(p, 2 p)}(\vec{r}) \phi_{n \kappa m}(\vec{r}) .
$$

Working along similar lines, one can derive a factorized form of the $A\left(e, e^{\prime} p\right)$ differential cross section [21],

$$
\frac{d^{5} \sigma}{d \epsilon^{\prime} d \Omega_{e^{\prime}} d \Omega_{N}}=\frac{k_{N} M_{A-1} M_{N}}{M_{A}}\left(f_{\text {rec }}^{e A}\right)^{-1} \sigma^{e p} S_{n \kappa} \rho_{n \kappa}^{D}\left(\vec{p}_{m}\right),
$$

where $\sigma^{e p}$ is the off-shell electron-proton cross section obtained from positive-energy projections. The distorted momentum distribution is defined as in Eq. (14) whereby $\widehat{\mathcal{S}}_{\mathrm{RMSGA}}^{(p, 2 p)}$ is replaced with $\widehat{\mathcal{S}}_{\mathrm{RMSGA}}^{\left(e, e^{\prime} p\right)}$ that adopts the form [17]

$$
\widehat{\mathcal{S}}_{\mathrm{RMSGA}}^{\left(e, e^{\prime} p\right)}(\vec{r})=\prod_{i=2}^{i=A} \int d \vec{r}_{i}\left|\phi_{\alpha_{i}}\left(\vec{r}_{i}\right)\right|^{2} \widehat{S}_{k 1}\left(\vec{r}, \vec{r}_{2}, \ldots, \vec{r}_{A}\right)
$$

\section{B. Relativistic multiple-scattering Glauber approximation}

The quantity $\delta(r, \theta) d r d \theta$ defined in Eq. (14) encodes the contribution from an infinitesimal interval $[r+d r, \theta+d \theta]$ to the cross section for a quasifree $p+A \rightarrow p+p+A-1$ process that leaves the residual nucleus in a hole state determined by the quantum numbers $(n \kappa m)$ [22]. The eikonal operator $\widehat{\mathcal{S}}_{\mathrm{RMSGA}}^{(p, 2 p)}(\vec{r})$ of Eq. (12) receives contributions from the impinging $\left(\widehat{S}_{p 1}\right)$ and the two ejected $\left(\widehat{S}_{k 1}, \widehat{S}_{k 2}\right)$ protons. In Refs. [17] and [23] we developed a relativized version of Glauber multiple-scattering theory. In this so-called RMSGA approach the eikonal phases are diagonal $2 \times 2$ matrices

$$
\begin{aligned}
\widehat{S}_{p 1}\left[\vec{r}(\vec{b}, z), \vec{r}_{2}, \ldots, \vec{r}_{A}\right] & =\prod_{j=2}^{j=A}\left[1-\Gamma\left(\vec{b}-\vec{b}_{j}\right) \theta\left(z_{j}-z\right)\right] \\
& =\exp i \chi\left[\vec{r}(\vec{b}, z), \vec{p}_{1}\right]
\end{aligned}
$$

For the profile functions $\Gamma_{p N}$ for proton-nucleon scattering we adopt the standard Gaussian parametrization

$$
\Gamma_{p N}(b)=\frac{\sigma_{p N}^{\mathrm{tot}}\left(1-i \epsilon_{p N}\right)}{4 \pi \beta_{p N}^{2}} \exp -\frac{b^{2}}{2 \beta_{p N}^{2}}
$$

where $\epsilon_{p N}, \beta_{p N}, \sigma_{p N}^{\text {tot }}$ have been determined from the database of proton-proton and proton-neutron cross sections [17].

It can be expected that the nucleon-nucleon interactions entering Eq. (18) by means of the profile function $\Gamma_{p N}$ will be subject to medium modifications [24,25]. Mechanisms such as Pauli blocking often lead to an effective reduction of the nucleon-nucleon cross sections in the medium. At higher energies, the effect of Pauli blocking is expected to become small. Another important source of medium effects in the treatment of ISI and FSI includes SRCs [26-28]. The SRCs are related to the finite size of the nucleons and the liquid properties of the nucleus. Indeed, the presence of a nucleon at some position $\vec{r}$ induces local fluctuations in the nuclear density. These local fluctuations, which go beyond the mean-field picture, can be included in the numerical evaluation of the operators $\widehat{\mathcal{S}}_{\mathrm{RMSGA}}^{\left(e, e^{\prime} p\right)}(\vec{r})$ and $\widehat{\mathcal{S}}_{\mathrm{RMSGA}}^{(p, 2 p)}(\vec{r})$, where $\vec{r}$ is the interaction point of the impinging beam. With Eq. (18), the $\widehat{\mathcal{S}}_{\text {RMSGA }}^{(p, 2 p)}(\vec{r})$ of Eq. (12) has a very intuitive interpretation: It represents the accumulated phase of one incoming and two outgoing waves, which are subject to a medium with $(A-1)$ gray disks characterized by the profile function $\Gamma_{p N}$ and distributed over the medium by a density distribution of the mean-field type $\prod_{i=2}^{i=A}\left|\phi_{\alpha_{i}}\left(\vec{r}_{i}\right)\right|^{2}$. The latter expression for the density distribution can be corrected for SRCs, by using the information that for a reaction to take place a nucleon should be present at the initial interaction point [29]. This can be achieved in the following way. First, the squared single-particle wave functions in the Eqs. (12) and (17) can be approximated by the one-body density of the target nucleus $\rho_{A}^{[1]}(\vec{r})$ [normalized as $\left.\int d \vec{r} \rho_{A}^{[1]}(\vec{r})=A\right]$ :

$$
\left|\phi_{\alpha_{i}}\left(\vec{r}_{i}\right)\right|^{2} \longrightarrow \frac{\rho_{A}^{[1]}\left(\vec{r}_{i}\right)}{A}=\frac{\sum_{i=1}^{i=A}\left|\phi_{\alpha_{i}}\left(\vec{r}_{i}\right)\right|^{2}}{A} .
$$

This substitution has a relatively minor impact on the computed effect of ISI/FSI in the RMSGA model [17]. Without any loss of generality the $\rho_{A}^{[1]}(\vec{r})$ can be replaced by the ratio of the two-body density $\rho_{A}^{[2]}$ [normalized as $\int d \vec{r}_{1} \int d \vec{r}_{2} \rho_{A}^{[2]}\left(\vec{r}_{1}, \vec{r}_{2}\right)=$ $A(A-1)]$ and the one-body density

$$
\rho_{A}^{[1]}\left(\vec{r}_{2}\right) \rightarrow \frac{A}{A-1} \frac{\rho_{A}^{[2]}\left(\vec{r}_{2}, \vec{r}\right)}{\rho_{A}^{[1]}(\vec{r})},
$$

where $\vec{r}$ is the coordinate of the probe-target interaction. For an uncorrelated two-body density one has

$$
\rho_{A, \text { uncorr. }}^{[2]}\left(\vec{r}_{1}, \vec{r}_{2}\right) \equiv \frac{A-1}{A} \rho_{A}^{[1]}\left(\vec{r}_{1}\right) \rho_{A}^{[1]}\left(\vec{r}_{2}\right),
$$

and Eq. (20) becomes trivial. One can implement the effect of central SRCs in the two-body density by adopting the following functional form [29,30]:

$$
\rho_{A, \text { corr. }}^{[2]}\left(\vec{r}_{1}, \vec{r}_{2}\right) \equiv \frac{A-1}{A} \gamma\left(\vec{r}_{1}\right) \rho_{A}^{[1]}\left(\vec{r}_{1}\right) \rho_{A}^{[1]}\left(\vec{r}_{2}\right) \gamma\left(\vec{r}_{2}\right) g\left(r_{12}\right),
$$

where $g\left(r_{12}\right)$ is the so-called Jastrow correlation function [31] and $\gamma(\vec{r})$ a function which guarantees the proper normalization of $\rho_{A}^{[2]}$. The $\gamma(\vec{r})$ can be numerically obtained as the solution of an integral equation. With the above expression for the 


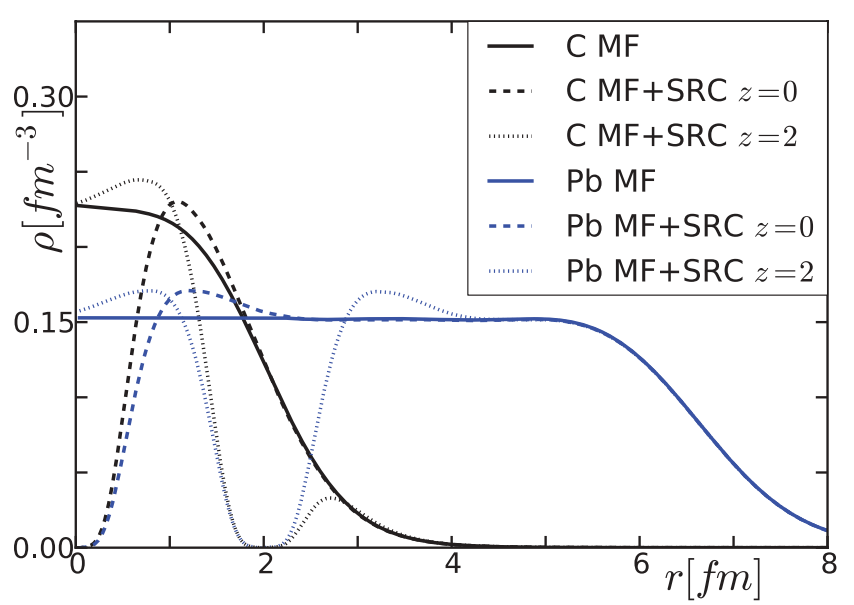

FIG. 2. (Color online) Comparison of the mean-field (MF) density $\rho_{A}^{[1]}(\vec{r})$ and the SRC-corrected effective density (denoted as $\mathrm{MF}+\mathrm{SRC}) \rho_{A}^{\text {eff }}\left[\vec{r}, \vec{r}^{\prime}=(0,0, z)\right]$ for the target nuclei ${ }^{12} \mathrm{C}$ and ${ }^{208} \mathrm{~Pb}$. The dashed (dotted) lines are for $z=0(z=2)$.

two-body density, Eq. (20) becomes

$$
\rho_{A}^{[1]}\left(\vec{r}_{2}\right) \rightarrow \gamma\left(\vec{r}_{2}\right) \rho_{A}^{[1]}\left(\vec{r}_{2}\right) \gamma(\vec{r}) g\left(\left|\vec{r}_{2}-\vec{r}\right|\right) \equiv \rho_{A}^{\text {eff }}\left(\vec{r}_{2}, \vec{r}\right) .
$$

From the above derivations it follows that the computation of ISI/FSI can be corrected for SRCs by replacing $\left|\phi_{\alpha_{i}}\left(\vec{r}_{i}\right)\right|^{2}$ with $\rho_{A}^{\text {eff }}\left(\vec{r}_{i}, \vec{r}\right) / A$ in Eqs. (12) and (17). The presence of a nucleon at the interaction point $\vec{r}$ induces local fluctuations in the probability distributions of the remaining $A-1$ nucleons. Within a radius $r \leqslant r_{N}$, where $r_{N}$ is the radius of a nucleon, of the initial interaction point, there will be a reduced probability of finding a nucleon to scatter from, whereas for $r \approx 2 r_{N}$ there will be an enhanced probability. The sole input required to determine $\rho_{A}^{\text {eff }}\left(\vec{r}_{2}, \vec{r}\right)$ from $\rho_{A}^{[1]}\left(\vec{r}_{2}\right)$ is the Jastrow correlation function $g\left(r_{12}\right)$. As this function is related to the short-range dynamics of nuclei it is considered "universal." We use a $g\left(r_{12}\right)$ that has a hard core extending over $0.8 \mathrm{fm}$ and a second bump with a peak at $r_{12} \approx 1.3 \mathrm{fm}$. With this choice for the $g\left(r_{12}\right)$ we obtained a fair description of the SRCs' contribution to the exclusive $\left(e, e^{\prime} p p\right)$ cross sections from ${ }^{12} \mathrm{C}$ [32] and ${ }^{16} \mathrm{O}$ [33]. In Fig. 2 we display for ${ }^{12} \mathrm{C}$ and ${ }^{208} \mathrm{~Pb}$ the effective density for a nucleon that is hit by an external probe in the center of the target nucleus $(z=0 \mathrm{fm})$ and in a more peripheral location along the $z$ axis at $2 \mathrm{fm}$ from the center $(z=2 \mathrm{fm})$ of the target nucleus. The SRCs induce a hole in the density at the position of the probe-target interaction point, and some enhancement at distances $\approx 1.5 \mathrm{fm}$ further away. The proposed method for implementing the effect of SRCs in Glauber calculations leads to effective densities (Fig. 2) that are qualitatively very similar to those produced in $a b$ initio calculations (see, for example, Fig. 1 of Ref. [34]). Therefore, we consider the proposed method of accounting for SRCs in the ISI/FSI calculation as realistic and efficient.

\section{RESULTS}

We now present the results of the numerical calculations for $\delta(r, \theta)$. The function $\delta(r, \theta)$ depends on the quantum
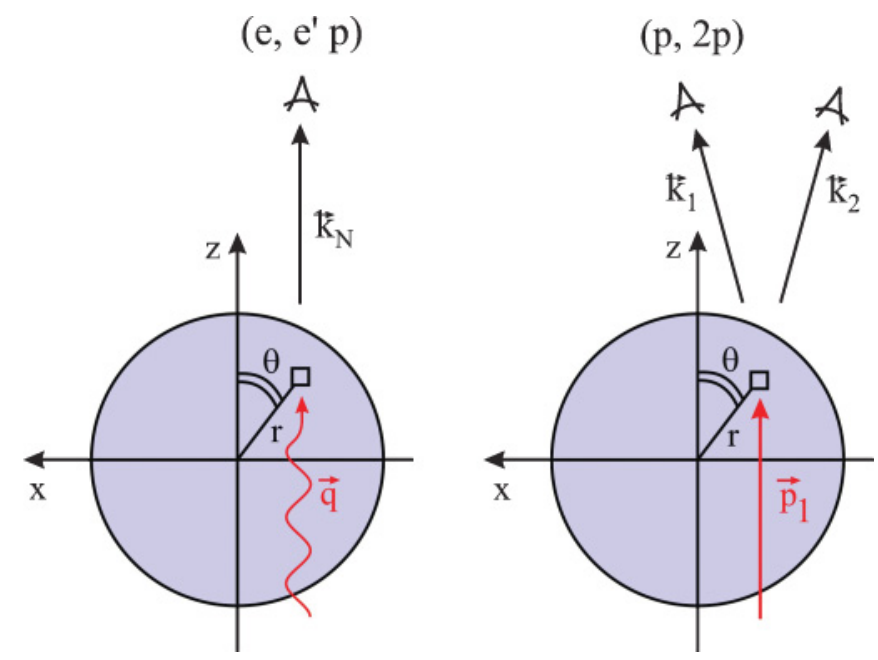

FIG. 3. (Color online) Schematic representation of the $A\left(e, e^{\prime} p\right)$ reaction in parallel kinematics and the $A(p, 2 p)$ reaction in coplanar and symmetric kinematics. The quantity $\delta(r, \theta) d r d \theta$ provides the contribution from the interval $[r+d r, \theta+d \theta]$ to the cross section.

numbers of the bound nucleon that collides with the proton or electron beam and the kinematics of the reaction. The considered $A\left(e, e^{\prime} p\right)$ and $A(p, 2 p)$ differential cross sections involve five kinematic variables. The presented investigations aim at determining global trends and choices with regard to the kinematics are in order. For the $A\left(e, e^{\prime} p\right)$ reaction we restrict ourselves to so-called parallel kinematics: the final nucleon is detected along the direction of the momentum transfer $\vec{q}$. For $A(p, 2 p)$ we consider coplanar and symmetric kinematics: the two ejected nucleons have equal kinetic energies $\left(\left|\vec{k}_{1}\right|=\left|\vec{k}_{2}\right|\right)$ and escape with equal opening angle but on opposite sides relative to the direction of the momentum transfer. The considered kinematics is illustrated in Fig. 3. We stress that in many respects there are strong analogies between the selected kinematics so that meaningful comparisons between $\left(e, e^{\prime} p\right)$ and $(p, 2 p)$ can be made.

The effect of ISI and FSI can be nullified by setting $\widehat{\mathcal{S}}_{\mathrm{RMSGA}}=1$ in Eqs. (12) and (17). Under those conditions one adopts the IA and all impinging and ejected nucleons are described by plane waves. Accordingly, we refer to the corresponding reaction model as the relativistic plane-wave impulse approximation (RPWIA). The difference between the RPWIA and RMSGA predictions for $\delta(r, \theta)$ can be exclusively attributed to nuclear attenuation. In the calculations including attenuation, we discriminate between the "RMSGA" and the "RMSGA + SRC" model variants. The latter includes the effect of SRCs in the modeling of the ISI/FSI.

In what follows we display $\left(e, e^{\prime} p\right)$ and $(p, 2 p)$ results for the target nuclei ${ }^{4} \mathrm{He},{ }^{12} \mathrm{C},{ }^{56} \mathrm{Fe}$, and ${ }^{208} \mathrm{~Pb}$ and use the relativistic $\sigma \omega$ model to determine their single-particle wave functions. We define the $z$ axis along the $\vec{q}$ and the $x z$ plane as the reaction plane. The ejected nucleons are detected in the forward direction. We start our discussions with the results for the medium-heavy target nucleus ${ }^{56} \mathrm{Fe}$.

In Fig. 4 we display the function $\delta(r, \theta)$ for proton knockout from the $1 s_{1 / 2}, 1 p_{3 / 2}, 1 d_{3 / 2}$, and $1 f_{7 / 2}$ orbits from ${ }^{56} \mathrm{Fe}$. We compare the $(p, 2 p)$ with the $\left(e, e^{\prime} p\right)$ result for an energy 

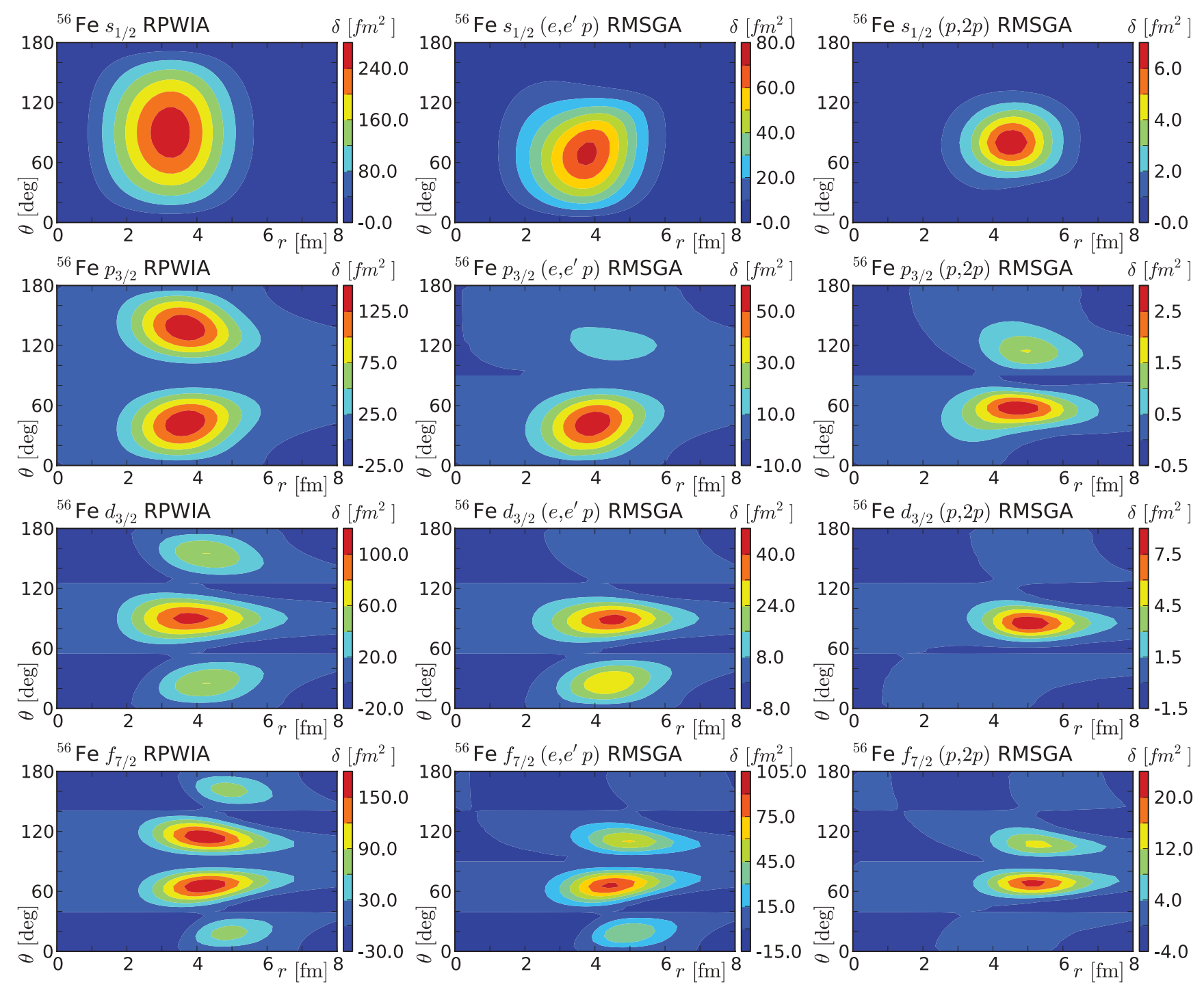

FIG. 4. (Color online) The function $\delta(r, \theta)$ for knockout from various shells in the ${ }^{56} \mathrm{Fe}$ target nucleus and ejected nucleon kinetic energies of $1.5 \mathrm{GeV}$. The $\left(e, e^{\prime} p\right)$ results are for parallel kinematics. For $(p, 2 p)$ coplanar and symmetric kinematics are considered. The magnitude of the momentum transfer $|\vec{q}|$ is adjusted so as to probe the maximum of the momentum distribution. This corresponds with $p_{m}=0 \mathrm{MeV}$ for the $1 s_{1 / 2}, p_{m}=105 \mathrm{MeV}$ for the $1 p_{3 / 2}, p_{m}=145 \mathrm{MeV}$ for the $1 d_{3 / 2}$, and $p_{m}=180 \mathrm{MeV}$ for the $1 f_{7 / 2}$. For the sake of reference, the measured proton root-mean-square radius in ${ }^{56} \mathrm{Fe}$ is $r_{\text {rms }} \approx 3.75 \mathrm{fm} \mathrm{[35].}$

transfer of $1.5 \mathrm{GeV}$ and conditions probing the maximum of the undistorted momentum distribution

$$
\rho_{n \kappa}\left(\vec{p}_{m}\right)=\sum_{s, m}\left|\int d \vec{r} \frac{e^{-i \vec{p}_{m} \cdot \vec{r}}}{(2 \pi)^{3 / 2}} \bar{u}\left(\vec{p}_{m}, s\right) \phi_{n \kappa m}(\vec{r})\right|^{2} .
$$

The $x$ axis is a symmetry axis for $\delta(r, \theta)$ in the RPWIA reaction picture. Indeed, in the absence of nuclear attenuation, the upper $\left(0^{\circ} \leqslant \theta \leqslant 90^{\circ}\right)$ and lower hemisphere $\left(90^{\circ} \leqslant \theta \leqslant\right.$ $\left.180^{\circ}\right)$ of the target nucleus equally contribute to the measured signals in the detectors and the $\delta(r, \theta)$ becomes equal for $\left(e, e^{\prime} p\right)$ and $(p, 2 p)$. In RPWIA the $\delta(r, \theta)$ reflects the symmetry imposed by the quantum numbers $(n \kappa)$. It is obvious that with increasing orbital angular momentum $l$ the detected signals are increasingly stemming from the peripheral areas of the target nucleus. The ISI and FSI have the strongest impact at the highest nuclear densities. This reflects itself in the fact that the largest values of $\delta(r, \theta)$ in the RMSGA model are shifted to larger values of $r$ in comparison with what one finds in RPWIA. As can be appreciated from Fig. 4 this shift is strongest for the deep-lying $1 s_{1 / 2}$ orbit and less pronounced for the valence $1 f_{7 / 2}$ orbit. Further, attenuation breaks the symmetry between the contribution from the upper and lower hemisphere as the first one is positioned closer to the detector(s). The degree of asymmetry between the contribution to the cross section from $(r, \theta)$ and $\left(r, 180^{\circ}-\theta\right)$ is a measure for the impact of attenuation. Another indicator is the ratio of the magnitude of RMSGA to the RPWIA prediction for $\delta(r, \theta)$. Loosely speaking, one could interpret this ratio as the fraction of the available signal in the target nucleus at some position $(r, \theta)$ that can withstand the attenuating nuclear medium and makes it to the detectors. Obviously, the induced 

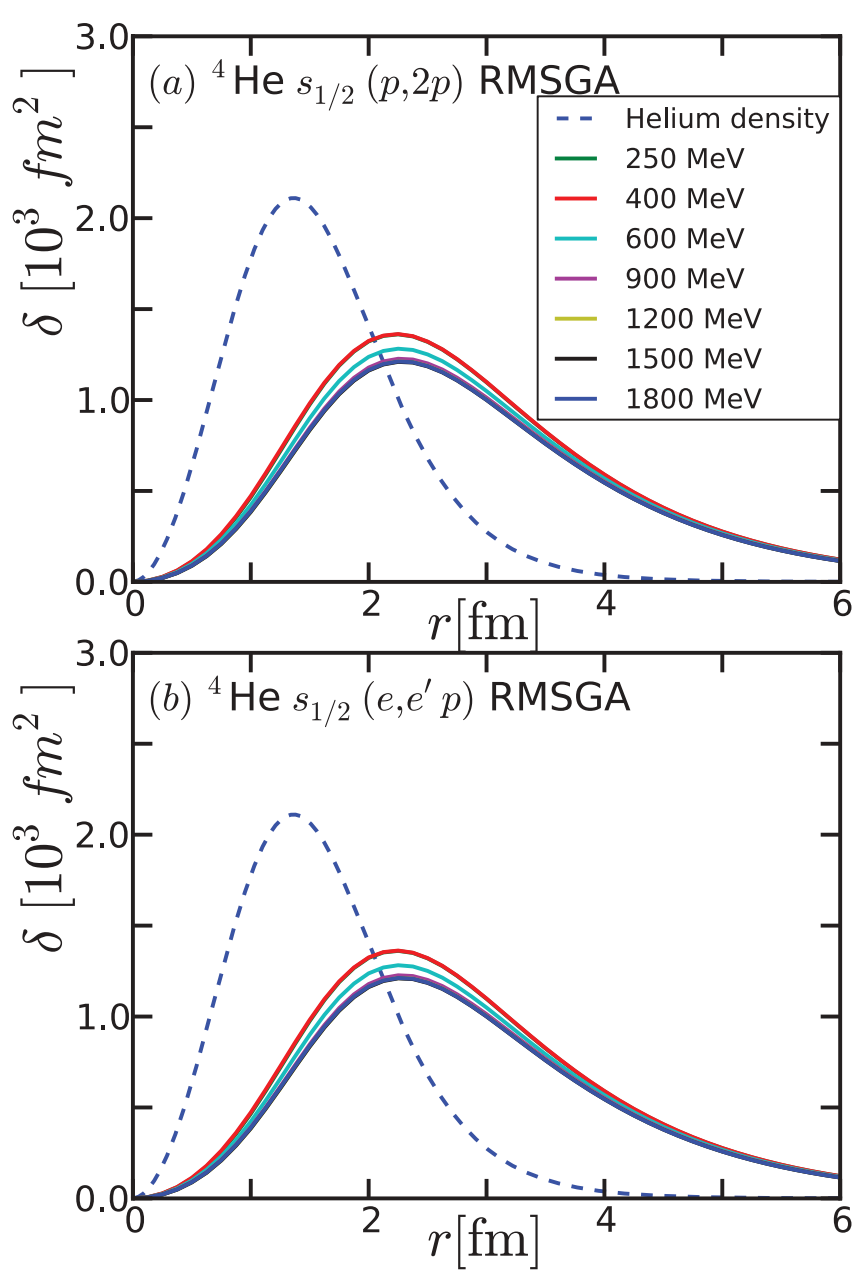

FIG. 5. (Color online) The energy dependence of the radial reaction probability densities $\delta(r)=\int d \theta \delta(r, \theta)$. We consider proton knockout from ${ }^{4} \mathrm{He}$ at ejected proton kinetic energies of 250, 400, $600,900,1200,1500$, and $1800 \mathrm{MeV}$. The $\left(e, e^{\prime} p\right)[(p, 2 p)] \mathrm{RMSGA}$ results are for parallel (coplanar-symmetric) kinematics. For the sake of reference the $r^{2} \rho^{[1]}(r)$ for ${ }^{4} \mathrm{He}$ is shown (not to scale).

angular asymmetry, radial shift, and overall reduction occur for the $\delta(r, \theta)$ in both $\left(e, e^{\prime} p\right)$ and $(p, 2 p)$. All three effects, however, are far more pronounced for the $(p, 2 p)$ than for the corresponding $\delta(r, \theta)$ in $\left(e, e^{\prime} p\right)$. Further, one observes that the combined effect of ISI/FSI gradually diminishes as one moves from the deep-lying to the valence single-particle orbits.

The preceding discussion concerned one particular proton kinetic energy. Next, we report on the study of the energy dependence of the impact of nuclear attenuation. As a representative example, in Fig. 5 we show for $\left(e, e^{\prime} p\right)$ and $(p, 2 p)$ the radial reaction probability distribution $\delta(r)=\int d \theta \delta(r, \theta)$ for proton knockout from ${ }^{4} \mathrm{He}$ at kinetic energies ranging from 0.25 to $1.8 \mathrm{GeV}$. One observes a very soft energy dependence in the radial dependence and the magnitude of the $\delta(r)$. Therefore, we deem that the results for the average densities and radii that will be presented below and that are obtained at a specific kinetic energy of $1.5 \mathrm{GeV}$ can be considered as representative for nucleon knockout reactions with $T_{N} \gtrsim 250 \mathrm{MeV}$. In Figs. 5, 6, and 7 we have added the function $r^{2} \rho_{A}^{[1]}(r)$. The
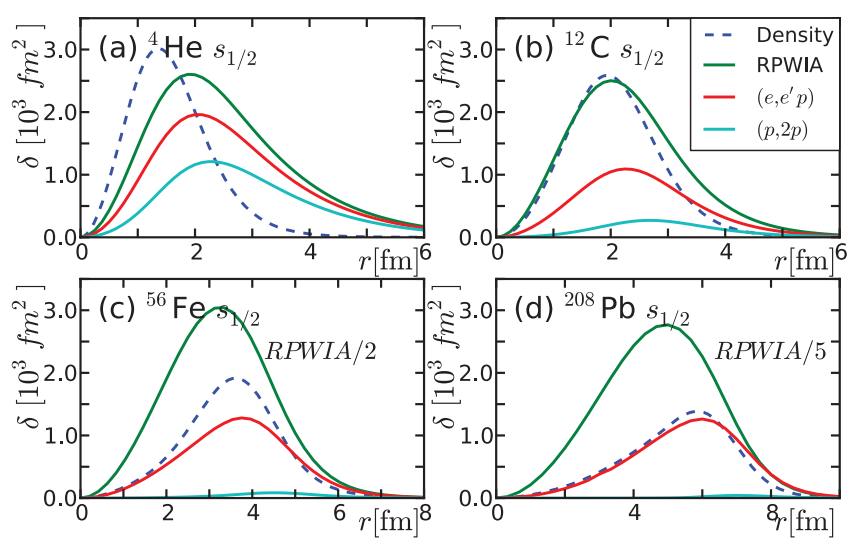

FIG. 6. (Color online) The target mass dependence of the radial reaction probability density $\delta(r)$. We consider proton knockout from the deep-lying $1 s_{1 / 2}$ level and ejected proton kinetic energies of $1500 \mathrm{MeV}$. The magnitude of the momentum transfer $|\vec{q}|$ is adjusted so as to probe the maximum of the momentum distribution $\left(p_{m}=0\right.$ $\mathrm{MeV})$. For the sake of reference we added $r^{2} \rho_{A}(r)$ for the various target nuclei (not to scale).

densities are computed with the basis of relativistic singleparticle wave functions which is also used in the reaction dynamics calculations. The densities are plotted not to scale and help in evaluating to what extent the knockout process succeeds in probing the bulk regions of the target nucleus.

We wish to investigate the target-mass dependence of $\delta(r, \theta)$. The results of Fig. 4 pointed toward a strong orbit dependence in the average radius and density that can be probed. We consider knockout from the deep-lying $1 s_{1 / 2}$ (Fig. 6) and one of the weakly bound orbits (Fig. 7) for the four representative target nuclei considered in this work. For ${ }^{208} \mathrm{~Pb}$ we opted for the $1 h_{11 / 2}$ valence orbit as it is representative for orbits with large orbital momentum. The $1 h_{11 / 2} \frac{11}{2}^{-}$hole state in ${ }^{207} \mathrm{Tl}$ is located at an excitation energy of $1.35 \mathrm{MeV}$. Results of the RPWIA and RMSGA calculations are contained in the Figs. 6 and 7. We compare the $\delta(r)$ for the $(p, 2 p)$ reaction with the $\left(e, e^{\prime} p\right)$ one. The RPWIA result for $\delta(r)$ is the reference figure of merit for knockout from a particular orbit. The mass dependence can
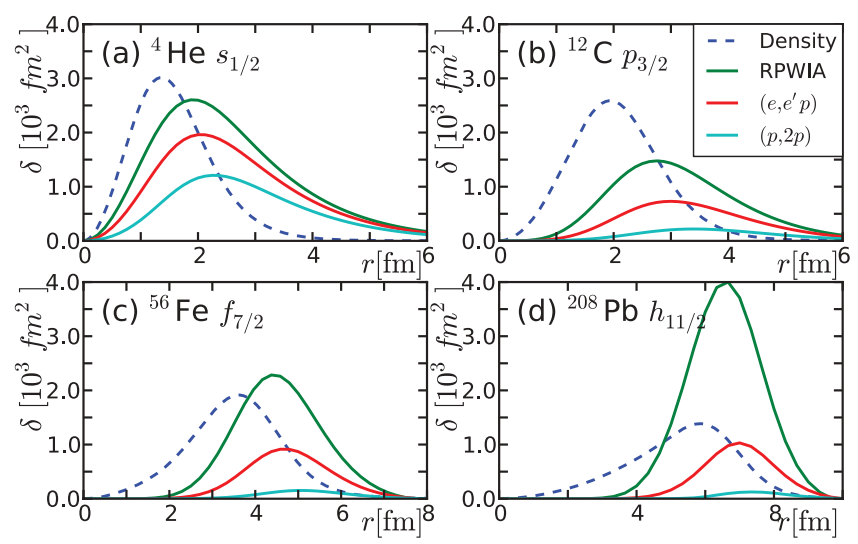

FIG. 7. (Color online) As in Fig. 6 but now for knockout from one of the valence orbits. 

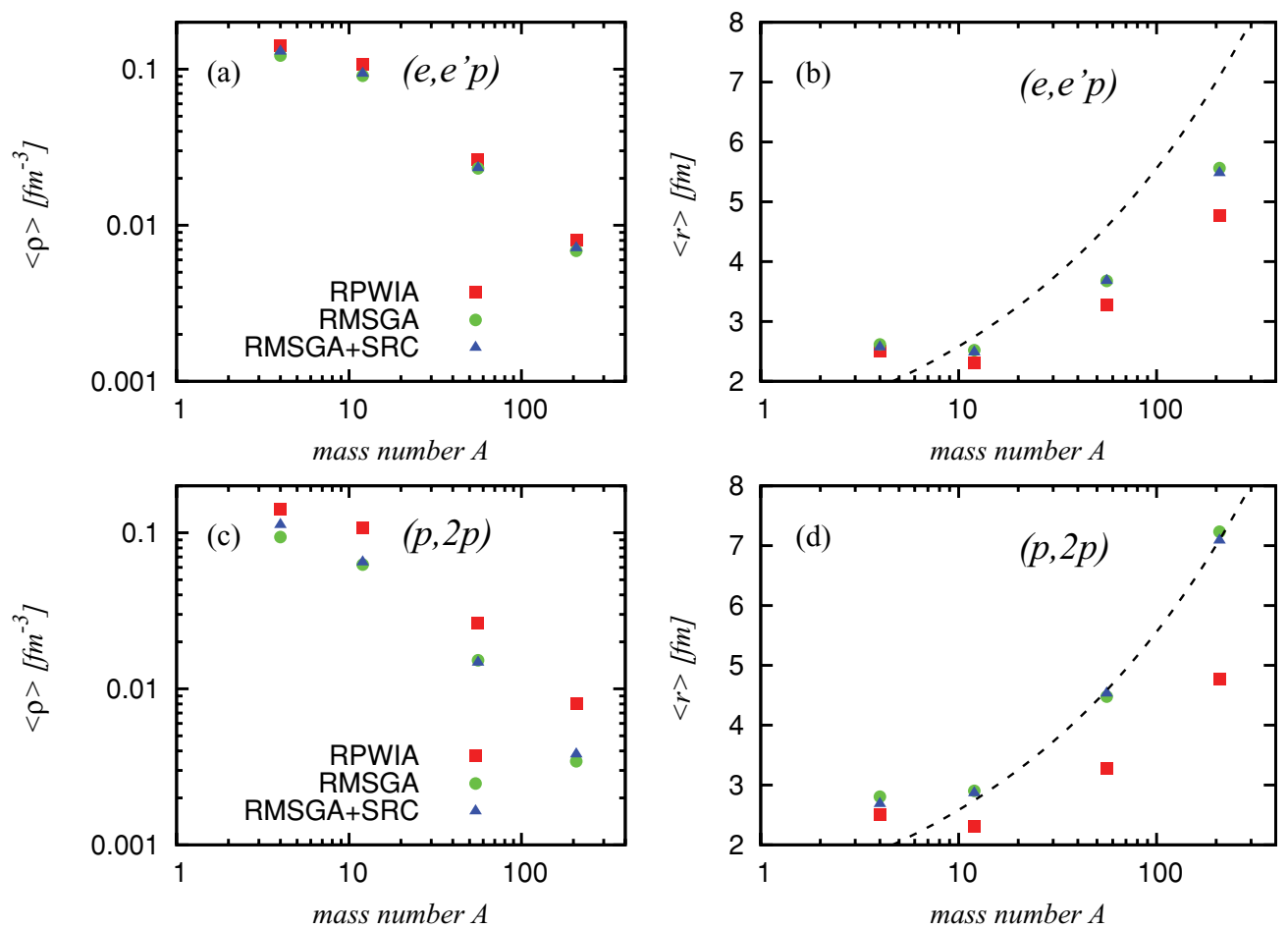

FIG. 8. (Color online) The target-mass dependence of the average density $\langle\rho\rangle$ and the average radius $\langle r\rangle$ that can be probed in single-nucleon knockout from the deep-lying $1 s$ shell. The kinematic conditions are those from Fig. 6 . The dotted line is $1.2 A^{1 / 3}$.

be best appreciated from the $1 s_{1 / 2}$ knockout results from Fig. 6. The most spectacular observation is the enormous decrease in the absolute value of the $\operatorname{RMSGA}(p, 2 p)$ radial reaction transition density for medium-heavy and heavy nuclei. For the $1 s_{1 / 2}$ orbit, attenuation sheds about $90 \%$ of the $(p, 2 p)$ RPWIA signal in ${ }^{12} \mathrm{C}$; for ${ }^{208} \mathrm{~Pb}$ this becomes more than $99 \%$.
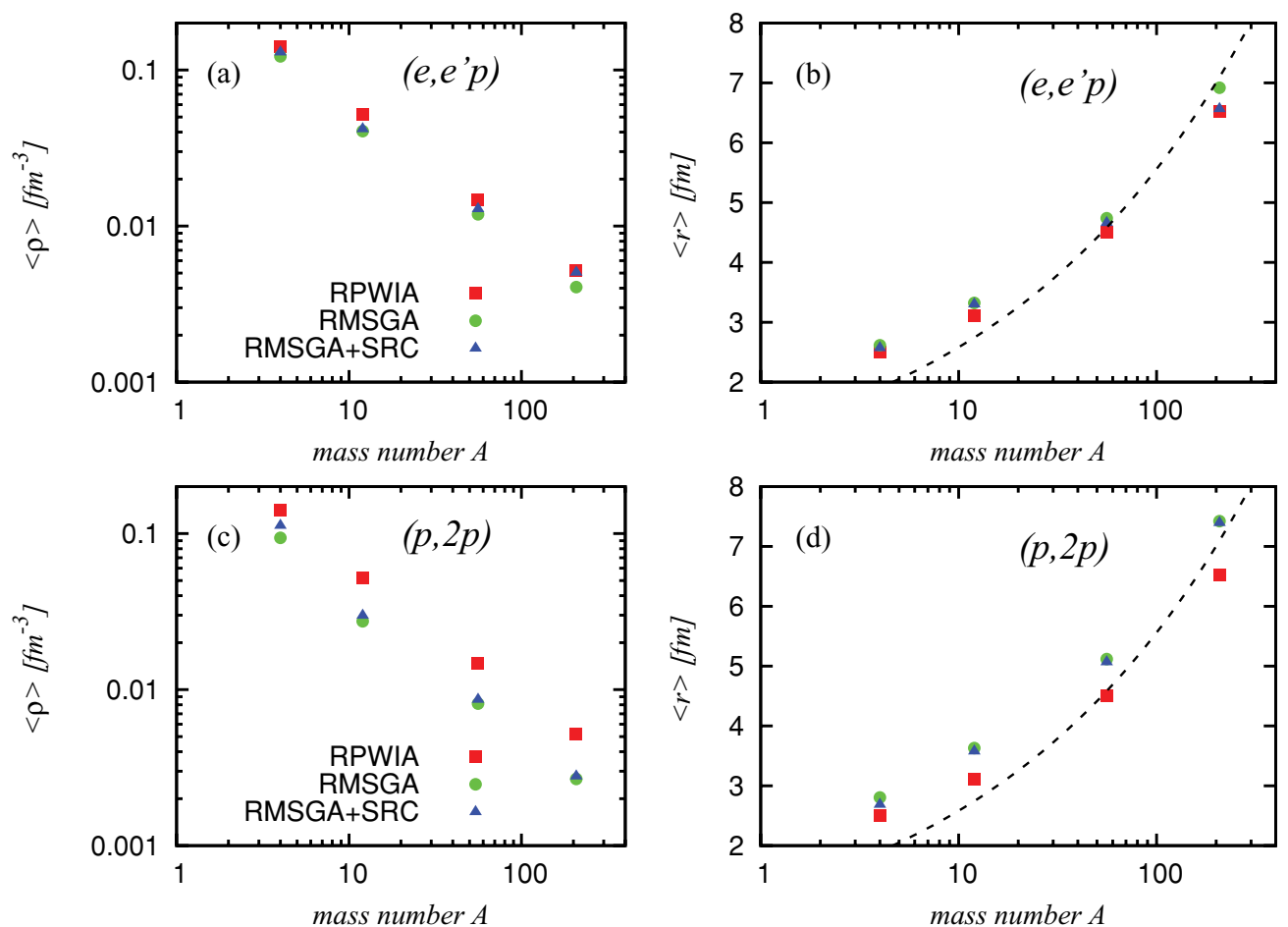

FIG. 9. (Color online) The target-mass dependence of the average density $\langle\rho\rangle$ and the average radius $\langle r\rangle$ that can be probed in single-nucleon knockout from the valence shell. The kinematic conditions are those from Fig. 7. The dotted line is $1.2 A^{1 / 3}$. 
It is clear that for any meaningful extraction of spectroscopic factors from $(p, 2 p)$ data the availability of a highly reliable reaction model is of the utmost importance. For knockout from the valence orbits (Fig. 7) the major fraction of the signal stems from the tail of the density distribution of the target nucleus. For the light nuclei, the $\left(e, e^{\prime} p\right)$ reaction performs only slightly better than $(p, 2 p)$ when it comes to probing the bulk regions. For the heavy nuclei, the growing role of attenuation makes the reaction probability density increasingly peripheral, and it is obvious that this primarily affects the $(p, 2 p)$.

To quantify the average density and radius that can be probed in the quasifree single-nucleon knockout reactions, we introduce the quantities [22,36]

$$
\begin{gathered}
\langle\rho\rangle=\frac{\int d r d \theta \rho_{A}^{[1]}(\vec{r}) \delta(r, \theta)}{\int d r d \theta \delta(r, \theta)}, \\
\langle r\rangle=\frac{\int d r d \theta r \delta(r, \theta)}{\int d r d \theta \delta(r, \theta)},
\end{gathered}
$$

where $\rho_{A}^{[1]}(\vec{r})$ is the density of the target nucleus. From the foregoing discussions we infer that for a given target nucleus $A$ and orbit, the energy dependence of the effective densities $\langle\rho\rangle$ and effective radius $\langle r\rangle$ is rather smooth. For that reason we stick with $T_{p}=1.5 \mathrm{GeV}$ and compile in Figs. 8 and 9 the $\langle\rho\rangle$ and $\langle r\rangle$ for the $\left(e, e^{\prime} p\right)$ and $(p, 2 p)$ results contained in Figs. 6 and 7. The RPWIA prediction for the average radius $\langle r\rangle$ increases with $A$ at a slightly softer rate than $A^{1 / 3}$. Note that even in the idealized attenuation-free world described by RWPIA, there is a strong mass dependence in the average density that can be probed in a quasifree nucleon knockout process. This strong mass dependence was exploited in Ref. [22] to probe the medium dependence of the $N N$ scattering amplitude. In comparing extracted information from $\left(e, e^{\prime} p\right)$ and $(p, 2 p)$ reactions, such as spectroscopic factors, through the mass table, it is often not realized that the reactions probe increasingly the target's surface region with growing $A$. For proton knockout from ${ }^{4} \mathrm{He}$, RPWIA predicts an average density $\langle\rho\rangle=0.85 \rho_{0}$, with the nuclear saturation density $\rho_{0}=0.16 \mathrm{fm}^{-3} \cdot \mathrm{In}^{208} \mathrm{~Pb}$ and knockout from the $1 h_{11 / 2}$ valence shell we find $\langle\rho\rangle=0.03 \rho_{0}$. All this is before correcting for FSI/ISI, which further reduces the $\langle\rho\rangle$.

For light nuclei the RPWIA and RMSGA predictions for $\langle r\rangle$ are close. For heavier nuclei a different story emerges. For knockout from the $1 s_{1 / 2}$ orbit in ${ }^{208} \mathrm{~Pb}$, for example, attenuation makes the $\langle r\rangle$ to grow quite dramatically. Indeed, for $(p, 2 p)$ $\left[\left(e, e^{\prime} p\right)\right]$ the RMSGA prediction is $\sim 2.5 \mathrm{fm}(\sim 0.9 \mathrm{fm})$ larger than the RPWIA value of $4.71 \mathrm{fm}$. For the valence states (Fig. 9) the increase in $\langle r\rangle$ is not larger than $0.4 \mathrm{fm}$ in $\left(e, e^{\prime} p\right)$. In $(p, 2 p)$ the gain in $\langle r\rangle$ is larger, but even in $\mathrm{Pb}$ it is smaller than $1 \mathrm{fm}$. As mentioned before, the $\langle\rho\rangle$ decreases monotonically from the lightest to the heavier nuclei. Attenuation makes that, in reality, smaller $\langle\rho\rangle$ will be probed. The effect varies from a loss of couple of percent to a loss in $\langle\rho\rangle$ of more than $50 \%$. In ${ }^{12} \mathrm{C}$, the $(p, 2 p)$ reaction from the $s 1 / 2$ orbit can effectively probe higher densities $\left(\langle\rho\rangle=0.39 \rho_{0}\right)$ than the $\left(e, e^{\prime} p\right)$ reaction from the valence $p_{3 / 2}$ orbit $\left(\langle\rho\rangle=0.25 \rho_{0}\right)$. For the ${ }^{12} \mathrm{C}(p, 2 p)$ reaction with knockout from the $s 1 / 2$ orbit the RMSGA prediction of $\langle\rho\rangle=0.39 \rho_{0}$ is comparable to the
DWIA result $\langle\rho\rangle=0.34 \rho_{0}$ of Ref. [36], which is obtained for $1 \mathrm{GeV}$ incoming protons. This illustrates the robustness of the results of this work.

The results for $\langle\rho\rangle$ and $\langle r\rangle$ in Figs. 8 and 9 allow one to estimate the role of SRCs in the modeling of the attenuation. In line with the observations of Refs. [26] and [30] the SRCs make the nucleus somewhat more transparent. This reflects itself in the fact that after including the SRCs the RMSGA predictions for $\langle\rho\rangle$ and $\langle r\rangle$ are shifting toward the RPWIA values. We wish to emphasize that the results of Figs. 8 and 9 refer to kinematics corresponding with the maximum of the single-particle momentum distributions. On the basis of the densities shown in Fig. 2 one may be tempted to expect rather spectacular effects from SRCs in the ISI/FSI. The effect of SRCs on the $\langle\rho\rangle$ and $\langle r\rangle$ is rather moderate owing to the fact that the ISI/FSI are long-ranged in the longitudinal direction and short-ranged in the transverse direction [26].

Up to this point we have evaluated quasifree processes for which the kinematics is tuned to probe the maximum of the momentum distribution. Now we turn to a study of the distorted momentum distribution $\rho_{n \kappa}^{D}\left(p_{m}\right)$ as a function of the missing momentum $p_{m}$. We stick with parallel kinematics for $\left(e, e^{\prime} p\right)$ and coplanar and symmetric kinematics in $(p, 2 p)$. The variation in $p_{m}$ is achieved by varying the kinetic energies of the ejectiles at a fixed value of the momentum transfer $\vec{q}$. We study knockout from the deep-lying $1 s_{1 / 2}$ level for the various nuclei to get some feeling about the mass dependence of the attenuation. As one can appreciate from Fig. 10 with growing $A$ the RPWIA and RMSGA predictions for $\rho_{n \kappa}\left(p_{m}\right)$ increasingly diverge. A similar remark applies to the $\rho_{n \kappa}\left(p_{m}\right)$ for $\left(e, e^{\prime} p\right)$ and $(p, 2 p)$. For the valence states (Fig. 11) one observes similar trends, though less pronounced. This can be easily understood by considering that they probe the peripheral areas of the target nucleus.

At large missing momenta $p_{m}$ the effect of the SRC corrections on the distorted momentum distributions can be extremely large [26]. Here we concentrate on low missing momenta and observe that SRCs make the nucleus more transparent for the emission of nucleons. This observation complies with the conclusions of Refs. [26] and [30]. The
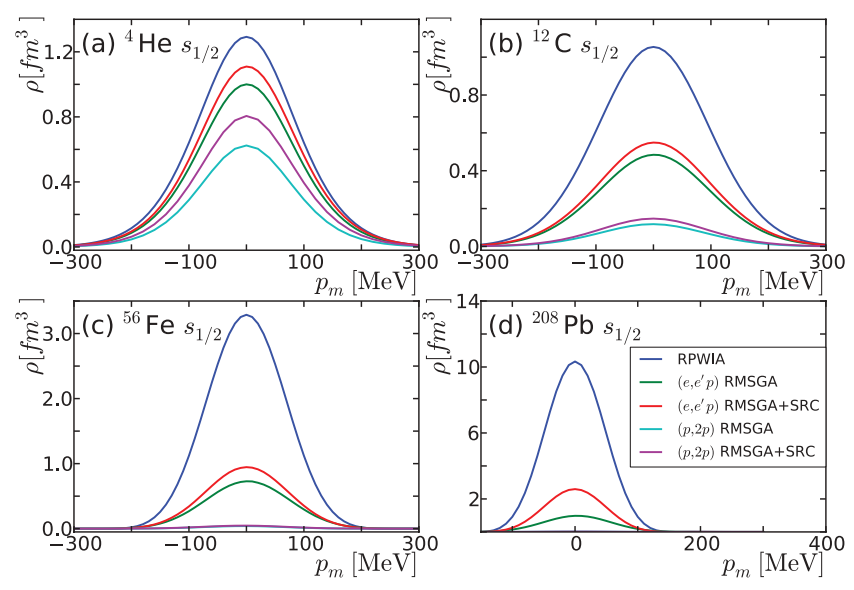

FIG. 10. (Color online) The RPWIA and RMSGA distorted momentum distribution for knockout from the deep-lying $1 s_{1 / 2}$ orbit. 

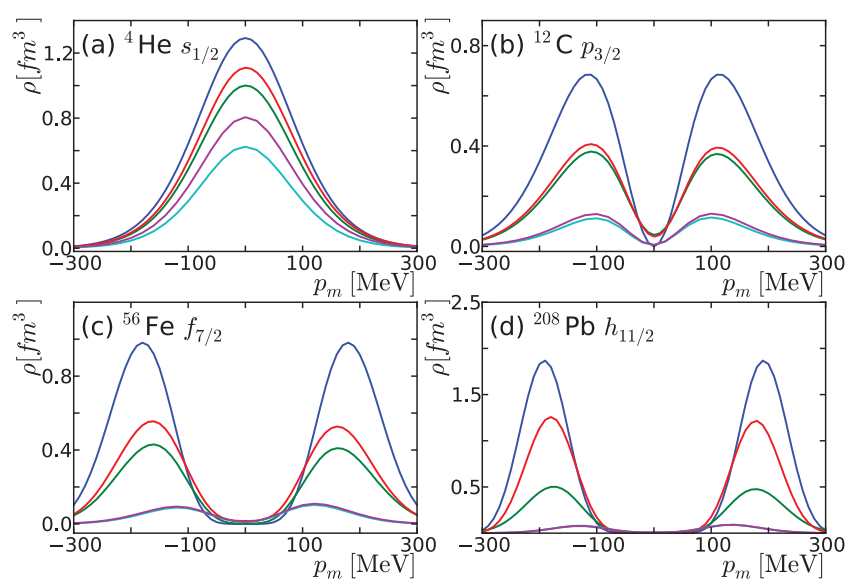

FIG. 11. (Color online) The RPWIA and RMSGA distorted momentum distribution for knockout from the valence shell. The curve coding is as in Fig. 10.

SRCs do not dramatically affect the $p_{m}$ dependence of the distorted momentum distributions at low $p_{m}$ but have an effect on its magnitude. This makes it of the utmost importance to use a correlated Glauber approach to extract precise information about the spectroscopic factors $S_{n \kappa}$.

\section{CONCLUSIONS}

In summary, we have used a relativistic framework to make a comparative and consistent study of the effective nuclear densities that can be probed in quasifree $(p, 2 p)$ and $\left(e, e^{\prime} p\right)$ reactions at high energies. We use relativistic single-particle wave functions from the $\sigma \omega$ model and a relativistic extension of Glauber multiple-scattering theory. Adopting the impulse and factorization factorization, both the $\left(e, e^{\prime} p\right)$ and the $(p, 2 p)$ cross sections are proportional to the distorted momentum distribution $\rho_{n \kappa}^{D}$, which include the effect of nuclear attenuation for the impinging and ejected nucleons. The $\rho_{n \kappa}^{D}$ reflects the effective momentum density for a bound nucleon with quantum numbers $n \kappa$ that is accessible for a certain reaction. We use this quantity as a tool to make a quantitative assessment of the role of nuclear attenuation for both types of reactions. Obviously, nuclear attenuation is a complicating factor in the extraction of nuclear-structure information from the measured single-nucleon knockout signals. Strong attenuation has a severe geometric influence as it makes the detected signal to carry little information about the nuclear interior. An interesting question, therefore, is how efficient $(p, 2 p)$ reactions are in probing the nuclear interior in comparison with the time-honored $\left(e, e^{\prime} p\right)$.

We have presented numerical results for knockout from one of the weakly bound levels and knockout from the deep-lying $1 s$ level from ${ }^{4} \mathrm{He},{ }^{12} \mathrm{C},{ }^{56} \mathrm{Fe}$, and ${ }^{208} \mathrm{~Pb}$. It emerges that to a remarkable extent the effect of the nuclear attenuation on the angular cross sections is independent of the energy of the initial and final protons. Accordingly, we have focused on one energy that we consider representative. The results for the average densities $\langle\rho\rangle$ which can be probed in quasifree singlenucleon knockout are collected and shown in Fig. 12. The
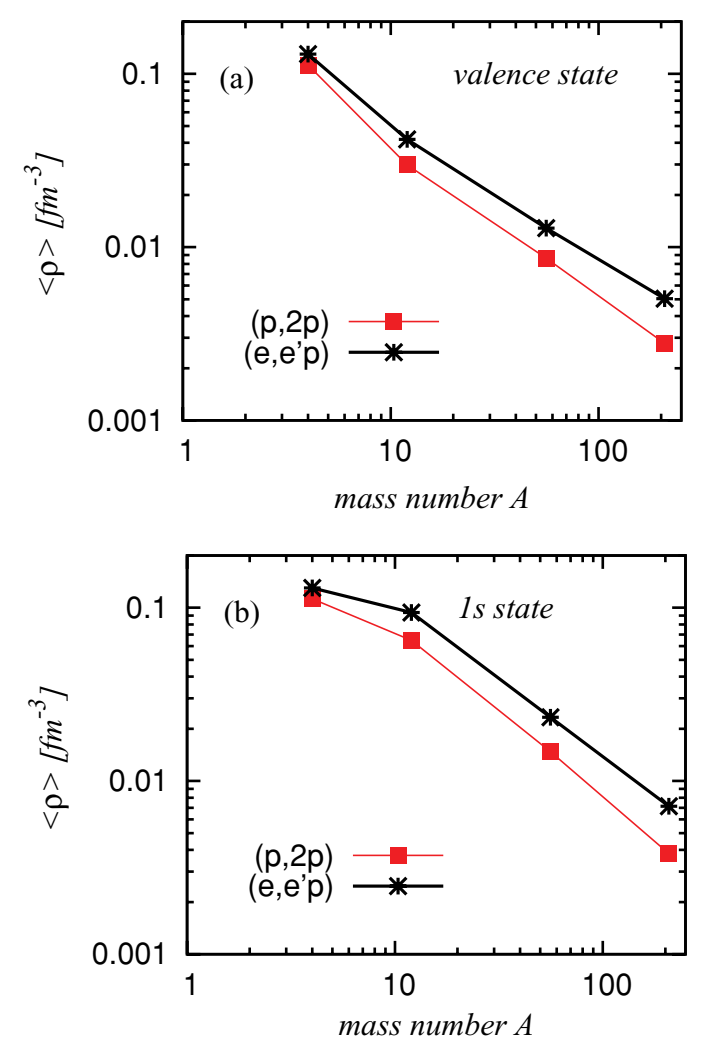

FIG. 12. (Color online) The predicted average density which can be probed in a quasifree nucleon knockout reaction. The results are obtained in the RMSGA framework and include the effect of SRCs.

$\langle\rho\rangle$ 's are very sensitive to the quantum numbers of the bound protons that collide with the probe. Further, there is strong target-mass dependence. Whereas in the lightest nuclei one can probe densities comparable to nuclear saturation density $\rho_{0}$, for a mid-heavy nucleus such as $\mathrm{Fe}$, this is of the order of $10 \%$ of $\rho_{0}$ and even smaller average densities are probed for a heavy nucleus such as $\mathrm{Pb}$. For light nuclei, the $(p, 2 p)$ and $\left(e, e^{\prime} p\right)$ reactions are comparably efficient in probing the nucleus' inner regions. In ${ }^{208} \mathrm{~Pb}$, the effect of attenuation is very dramatic for $(p, 2 p)$ and the average density which can actually be probed can be half of the $\left(e, e^{\prime} p\right)$ one.

Our model for the ISI and FSI implements the effect of short-range correlations. In line with previous studies we find that the SRCs tend to reduce the influence of attenuation. The SRCs moderately affect the average density and radius that can be probed. We do find, however, that after correcting for SRCs the cross sections can become substantially larger. The SRC corrections are particularly relevant for the heavier target nuclei and the channels that probe the inner-bound nucleons. As spectroscopic factors are typically obtained from the ratio of the measured and the computed distorted momentum distribution, the SRCs should become an essential ingredient of any model for nuclear attenuation.

\section{ACKNOWLEDGMENT}

This work was supported by the Fund for Scientific Research Flanders. 
[1] J. Kelly, Adv. Nucl. Phys. 23, 75 (1996).

[2] V. R. Pandharipande, I. Sick, and P. K. A. d. Huberts, Rev. Mod. Phys. 69, 981 (1997).

[3] L. Lapikas, Nucl. Phys. A 553, 297 (1993).

[4] R. J. Furnstahl and H. W. Hammer, Phys. Lett. B 531, 203 (2002).

[5] A. M. Mukhamedzhanov and A. S. Kadyrov, Phys. Rev. C 82, 051601 (2010).

[6] G. Jacob and T. A. J. Maris, Rev. Mod. Phys. 45, 6 (1973).

[7] H. Geissel et al., Nucl. Instrum. Methods Phys. Res. 204, 71 (2003).

[8] L. Chulkov et al., Nucl. Phys. A 759, 43 (2005).

[9] A. Gade et al., Phys. Rev. C 77, 044306 (2008).

[10] T. Kobayashi et al., Nucl. Phys. A 805, 431c (2008).

[11] T. Aumann, Prog. Part. Nucl. Phys. 59, 3 (2007).

[12] O. Benhar, S. Fantoni, N. Nikolaev, J. Speth, and B. Usmani, A. A. Zakharov, Z. Phys. A 355, 267 (1996).

[13] R. D. Amado, J. Piekarewicz, D. A. Sparrow, and J. A. McNeil, Phys. Rev. C 29, 936 (1984).

[14] P. Lava, M. C. Martinez, J. Ryckebusch, J. A. Caballero, and J. M. Udias, Phys. Lett. B 595, 177 (2004).

[15] G. C. Hillhouse and T. Noro, Phys. Rev. C 74, 064608 (2006).

[16] B. Van Overmeire, W. Cosyn, P. Lava, and J. Ryckebusch, Phys. Rev. C 73, 064603 (2006).

[17] J. Ryckebusch, D. Debruyne, P. Lava, S. Janssen, B. Van Overmeire, and T. Van Cauteren, Nucl. Phys. A 728, 226 (2003).

[18] G. D. Alkhazov, S. L. Belostotsky, and A. A. Vorobev, Phys. Rep. 42, 89 (1978).

[19] S. Jeschonnek and J. W. Van Orden, Phys. Rev. C 78, 014007 (2008).
[20] W. Cosyn and J. Ryckebusch, Phys. Rev. C 80, 011602 (2009).

[21] J. A. Caballero, T. W. Donnelly, E. Moya de Guerra, and J. M. Udias, Nucl. Phys. A 632, 323 (1998).

[22] K. Hatanaka et al., Phys. Rev. Lett. 78, 1014 (1997).

[23] B. Van Overmeire and J. Ryckebusch, Phys. Lett. B 650, 337 (2007).

[24] G. C. Hillhouse, B. I. S. Vander Ventel, S. M. Wyngaardt, and P. R. De Kock, Phys. Rev. C 57, 448 (1998).

[25] C. A. Bertulani and C. De Conti, Phys. Rev. C 81, 064603 (2010).

[26] A. Bianconi, S. Jeschonnek, N. N. Nikolaev, and B. G. Zakharov, Nucl. Phys. A 608, 437 (1996).

[27] M. Alvioli, C. Ciofi degli Atti, I. Marchino, V. Palli, and H. Morita, Phys. Rev. C 78, 031601 (2008).

[28] T.-S. H. Lee and G. A. Miller, Phys. Rev. C 45, 1863 (1992).

[29] W. Cosyn, M. C. Martinez, and J. Ryckebusch, Phys. Rev. C 77, 034602 (2008), arXiv:0710.4837.

[30] S. Frankel, W. Frati, and N. Walet, Nucl. Phys. A 580, 595 (1994).

[31] R. Roth, T. Neff, and H. Feldmeier, Prog. Part. Nucl. Phys. 65, 50 (2010).

[32] K. I. Blomqvist et al., Phys. Lett. B 421, 71 (1998).

[33] J. Ryckebusch and W. Van Nespen, Eur. Phys. J. A 20, 435 (2004).

[34] C. Ciofi degli Atti, arXiv:1011.5963.

[35] R. Peterson, H. Theissen, and W. Alston, Nucl. Phys. A 153, 610 (1970).

[36] V. A. Andreev et al., Phys. Rev. C 69, 024604 (2004). 